\title{
Attenuated Circadian Rhythms in Mice Lacking the Prokineticin 2 Gene
}

\author{
Jia-Da Li, ${ }^{1}$ Wang-Ping Hu, ${ }^{1}$ Lisa Boehmer, ${ }^{2}$ Michelle Y. Cheng, ${ }^{1}$ Alex G. Lee, ${ }^{1}$ Alexander Jilek, ${ }^{3}$ Jerome M. Siegel, ${ }^{2}$ and \\ Qun-Yong Zhou ${ }^{1}$ \\ ${ }^{1}$ Department of Pharmacology, University of California, Irvine, Irvine, California 92697, ${ }^{2}$ Neurobiology Research 151A3, Veterans Administration Greater \\ Los Angeles Healthcare System Sepulveda, Department of Psychiatry and Brain Research Institute, University of California, Los Angeles, School of \\ Medicine, North Hills, California 91343, and 'Institute of Organic Chemistry, Johannes-Kepler-University, 4040 Linz, Austria
}

Circadian clocks drive daily rhythms in virtually all organisms. In mammals, the suprachiasmatic nucleus (SCN) is recognized as the master clock that synchronizes central and peripheral oscillators to evoke circadian rhythms of diverse physiology and behavior. How the timing information is transmitted from the SCN clock to generate overt circadian rhythms is essentially unknown. Prokineticin 2 (PK2), a clock-controlled gene that encodes a secreted protein, has been indicated as a candidate SCN clock output signal that regulates circadian locomotor rhythm. Here we report the generation and analysis of PK2-null mice. The reduction of locomotor rhythms in PK2-null mice was apparent in both hybrid and inbred genetic backgrounds. PK2-null mice also displayed significantly reduced rhythmicity for a variety of other physiological and behavioral parameters, including sleep-wake cycle, body temperature, circulating glucocorticoid and glucose levels, as well as the expression of peripheral clock genes. In addition, PK2-null mice showed accelerated acquisition of food anticipatory activity during a daytime food restriction. We conclude that PK2, acting as a SCN output factor, is important for the maintenance of robust circadian rhythms.

Key words: circadian rhythm; prokineticin 2; knock-out; suprachiasmatic nucleus; sleep; locomotor

\section{Introduction}

In mammals, the pacemaker that drives circadian rhythms resides in the suprachiasmatic nucleus (SCN) of the anterior hypothalamus (Moore, 1997). Multiple interlocked transcriptional and translational loops consisting of a number of clockwork genes underlie the molecular mechanisms for the SCN clock (Buijs and Kalsbeek, 2001; Reppert and Weaver, 2002; Hastings and Herzog, 2004; Lowrey and Takahashi, 2004). Whereas diverse rhythms of physiology and behavior such as locomotor activity, sleep and wakefulness, body temperature, and energy metabolism are under the control of SCN, the primary neuronal targets of the SCN clock are limited (Watts and Swanson, 1987; Watts et al., 1987). Thus, to gain a better understanding of how the SCN controls circadian functions, it is critical to identify the signaling molecules that link SCN and these SCN primary efferent nuclei.

To date, several diffusible molecules have been suggested as candidate SCN output factors (Jin et al., 1999; Cheng et al., 2002; Kraves and Weitz, 2006). Vasopressin (AVP), a neuropeptide derived from a clock-controlled gene, may regulate circadian

Received Aug. 23, 2006; revised Sept. 27, 2006; accepted 0ct. 2, 2006.

This work was supported in part by National Institutes of Health-National Institute of Mental Health Grant NIMH067753. We thank Chongwei Wen and Chengkang Zhang for help with mouse genotyping and Eric L. Bittman, Alastair V. Ferguson, Kwan L. Ng, and Katherine J. Burton for critical comments on this manuscript.

Correspondence should be addressed to Qun-Yong Zhou, Department of Pharmacology, University of California, Irvine, Irvine, CA 92697. E-mail: qzhou@uci.edu.

DOI:10.1523/JNEUROSCI.3679-06.2006

Copyright $\odot 2006$ Society for Neuroscience $\quad$ 0270-6474/06/2611615-09\$15.00/0 rhythm of endocrine activity (Kalsbeek et al., 1996; Jin et al., 1999). Cardiotrophin-like cytokine (CLC) may be involved in the regulation of circadian locomotor rhythm, because intracerebroventricular delivery of CLC suppresses the locomotor activity in rodents, and the mRNA level of CLC displays temporal oscillation in the SCN (Kraves and Weitz, 2006). In addition, the nuclear proteins albumin D-element binding protein (DBP) and Per3 (Period 3) have also been implicated in SCN output (LopezMolina et al., 1997; Bae et al., 2001), although how they are linked to diffusible SCN molecules is still unclear.

We have shown previously that prokineticin 2 (PK2) is a candidate output molecule for the SCN clock (Cheng et al., 2002, 2005). PK2 belongs to a pair of unique cysteine-rich secreted proteins that also regulate other biological processes (LeCouter et al., 2001; Li et al., 2001; $\mathrm{Ng}$ et al., 2005). Multiple lines of evidence support the candidate role of PK2 in the transmission of circadian locomotor rhythm from the SCN clock. The mRNA expression of PK2 in the SCN displays drastic clockwork-dependent circadian oscillation under light/dark and constant dark conditions (Cheng et al., 2002; Lambert et al., 2005) Correlative in vitro studies have supported $P K 2$ as a first-order clock-controlled gene, with its expression controlled by Clock and Bmal1 (brain and muscle Arnt-like protein 1) acting on the E-boxes in its promoter. Moreover, the receptor for PK2 is expressed in virtually all known primary SCN targets (Cheng et al., 2002), a unique feature among the putative output signals. Intracerebroventricular delivery of recombinant PK2 suppresses the nocturnal wheel-running activity, when endogenous PK2 level is minimal (Cheng et al., 2002, 
2005). To gain insight into the physiological role of PK2 in the circadian rhythm, we generated mutant mice $\left(P \mathrm{~K}^{-/-}\right)$with the $P K 2$ gene disrupted by homologous recombination. A series of circadian-related behavioral, physiological, and molecular examinations on $P K 2^{-1-}$ mice revealed that PK2 plays an important role in the maintenance of robust circadian rhythms.

\section{Materials and Methods}

Generation of PK2-deficient mice. A targeting vector was constructed with phosphoglycerate kinase (pGK)-neomycin (Neo) as the positive selection marker and $\mathrm{pGK}-\mathrm{TK}$ as the negative selection marker to delete a 2.5 $\mathrm{kb}$ fragment including exon 1 and exon 2 . A $2.8 \mathrm{~kb}$ SacI-MluI fragment and a $4.3 \mathrm{~kb} \mathrm{XbaI}$ fragment were used as homologous fragments. A luciferase reading frame was inserted between the $5^{\prime}$ homology region and the Neo gene to result in the final construct shown in Figure $1 \mathrm{~A}$. The targeting construct was linearized with NotI and introduced into 129/Ola embryonic stem (ES) cells by electroporation. Genomic DNA was extracted from G418-resistant ES clones, digested with EcoRI and BamHI, and subjected to Southern blot hybridization. ES clones containing the disrupted $P K 2$ gene were injected into C57BL/6J blastocyst and transferred into pseudopregnant female recipients. Chimeric males were bred to C57BL6/J females. The heterozygous males and females (F1) were crossed to produce mice homozygous for the targeted allele $\left(P K 2^{-/-}\right)$. Genotyping of mice was done by both Southern blot hybridization and a three-primer PCR. After verifying the reliability of PCR genotyping, all routine screening was done by PCR. Mice used in the experiments were on C57BL/6 × 129/Ola hybrid background or a C57BL/6 inbred background (more than seven generations of backcrossing), 3-7 months of age, and mixed males and females, except for the experiment of measuring corticosterone and glucose levels, in which only males were used. All procedures regarding the care and use of animals are in accordance with institutional guidelines.

Measurement of the voluntary and spontaneous locomotor activities. Mice were individually housed within cages equipped with running wheels and were allowed to have access to food and water ad libitum. Mice were entrained to an initial $12 \mathrm{~h}$ light/dark (LD) cycle. After 3 weeks of activity recording in the LD cycle, the mice were placed in constant darkness with dim red light (DD) for 4-8 weeks for the assessment of endogenous period. The free-run period and fast Fourier transformation (FFT) were analyzed using ClockLab software (Actimetrics, Evanston, IL) in the Matlab (MathWorks, Natick, NA) environment (Bunger et al., 2000). The free-run period was measured by a $\chi^{2}$ periodogram from days 10 through 25 under DD. The daily revolution and FFT was determined by analyzing days 5 through 20 under LD and days 10 through 25 under DD.

For measurement of spontaneous activity, animals were housed in their home cages, which were placed in locomotor boxes equipped with 32 infrared emitters and receivers in two directions (Med Associates, St. Albans, VT). Spontaneous activity was defined as the number of infrared beam breaks per unit time.

Core body temperature monitoring. A radio transmitter device (G2 Emitter; Mini-Mitter, Sunriver, OR) used to measure body temperature was implanted in the abdominal cavity by sterile technique under general anesthesia (Nagashima et al., 2005). Body temperature was recorded by a receiver board (ER-4000 energizer receiver) underneath the cage and were stored in a personal computer every $5 \mathrm{~min}$. The mice were allowed to recover for at least 2 weeks before the experiments. The ambient temperature was $21^{\circ} \mathrm{C}$.

Recording and analysis of sleep-wake cycle. Electroencephalogram (EEG) and electromyogram (EMG) signals were recorded from wildtype (WT) and $P K 2^{-/-}$mice using a lightweight implant and cabling procedure as described previously (Naylor et al., 2000). After surgery, mice were allowed to recover for at least $7 \mathrm{~d}$ and then allowed to adapt for at least $7 \mathrm{~d}$ after the connection of a lightweight cable. EEG/EMG signals were collected using a Grass Instruments (Quincy, MA) model 15A94 Quad alternating current amplifier and digitized at a sampling rate of 256 $\mathrm{Hz}$. The signals were digitally filtered (EEG, $0.3-30 \mathrm{~Hz}$; EMG, 2-100 Hz) and semiautomatically scored in $4 \mathrm{~s}$ epochs as wake, nonrapid eye movement (NREM), or rapid eye movement (REM) sleep using SleepSign sleep scoring software (Kissei Comtec America, Irvine, CA). The total sleep is the sum of NREM and REM sleep. The preliminary scoring was visually inspected and corrected when appropriate.

Determination of corticosterone and glucose levels. Male wild-type and $P K 2^{-1-}$ mice were housed individually before collection of blood samples. Blood samples were collected by retro-orbital eye bleeding within $45 \mathrm{~s}$ of initial disturbance of the cages. Glucose levels were measured with OneTouch UltraSmart glucose meters (Lifescan, Milpitas, CA) from freshly collected blood samples. Plasma corticosterone levels were determined using radioimmunology assay kit (ICN Biomedicals, Costa Mesa, CA) according to the instructions.

Analysis of gene expression. Mice were killed every $4 \mathrm{~h}$ under a dim red light via cervical dislocation beginning at $49 \mathrm{~h}$ in constant darkness. Brains and peripheral tissues were quickly dissected and stored at $-80^{\circ} \mathrm{C}$ until use. In situ hybridization was performed on coronal sections as described previously (Cheng et al., 2002). For liver gene expression analysis, total liver RNA was isolated using RNeasy kit (Qiagen, Valencia, CA). Three micrograms of total RNA was reversed transcribed using Superscript II reverse transcriptase (Invitrogen, Carlsbad, CA), and $20 \mathrm{ng}$ of total RNA equivalents were then analyzed by a TaqMan technology in an ABI PRISM 7000 detection system (PE Applied Biosystems, Foster City, CA). The relative levels of Bmall and Dbp mRNAs were normalized to the corresponding glyceraldehyde-3-phosphate dehydrogenase levels. The primers and probes were designed as described previously (Bunger et al., 2000).

Restricted feeding. Mice were housed under LD with lights on at 6:00 A.M. and lights off at 6:00 P.M. The spontaneous activity of mice was monitored by counting infrared beam breaks. Food and water were supplied ad libitum for $6 \mathrm{~d}$. During restricted feeding (RF), food pellets were only available from Zeitgeber time 3 (ZT 3) to ZT 7 (9:00 A.M. to 1:00 P.M.), whereas water was supplied ad libitum. The food anticipatory activity (FAA) was defined as the percentage of activity occurred $3 \mathrm{~h}$ before the availability of food (6:00 A.M. to 9:00 A.M.) to the activity of previous 24 h (Dudley et al., 2003; Pitts et al., 2003).

Statistical analyses. A repeated-measures ANOVA followed by unpaired $t$ test was used to analyze the data for differences between genotypes. All statistical analysis was performed using Prism 4.4 (GraphPad Software, San Diego, CA).

\section{Results}

\section{Generation of $P K 2^{-/-}$mice}

To elucidate the physiological function of PK2, we generated $P K 2^{-/-}$mice. A targeting vector with exon 1 and 2 of the $P K 2$ gene replaced by a luciferase gene and a pGK-Neo cassette was constructed (Fig. 1A). The construct was transfected into 129/ Ola embryonic stem cells and clones that underwent homologous recombination were used to generate chimeric mice. Intercrossing of heterozygous mice produced $\sim 1500$ offspring with a distorted Mendelian distribution $\left(30.6 \% P K 2^{+/+}, 60.0 \% P K 2^{+/-}\right.$, and $9.4 \% P K 2^{-/-}$, respectively; $p<0.01, \chi^{2}$ test) when genotyped at 3 weeks after birth (Fig. $1 B, C$ ). The survival rate of $P K 2^{-1-}$ mice in the $\mathrm{C} 57 \mathrm{BL} / 6$ genetic background (more than seven generations of backcrossing) was further reduced. Additional analysis indicated that the lethality of $\mathrm{PK}^{-/-}$mice occurred postnatally, because the embryos from embryonic day 18.5 displayed the expected Mendelian ratio (data not shown). After weaning, $P K 2^{-1-}$ mice did not exhibit additional apparent morbidity. Deficiency of the $P K 2$ gene in $P K 2^{-/-}$mice was confirmed by in situ hybridization on brain sections. As shown in Figure 1, $D$ and $E$, no $P K 2$ hybridization signal was detected in the SCN of $P K 2^{-1-}$ mice collected over a $24 \mathrm{~h}$ period.

\section{Reduced circadian rhythms of locomotor activity in $P K 2^{-/-}$mice}

Locomotor activity, a commonly used index for circadian rhythmicity, can be monitored by either running wheels (e.g., volun- 


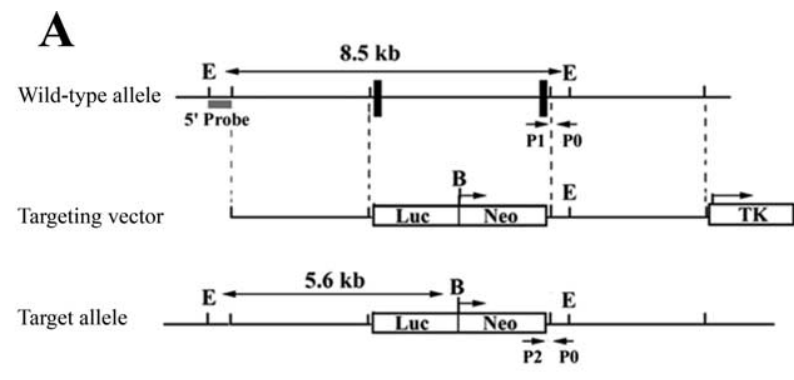

B
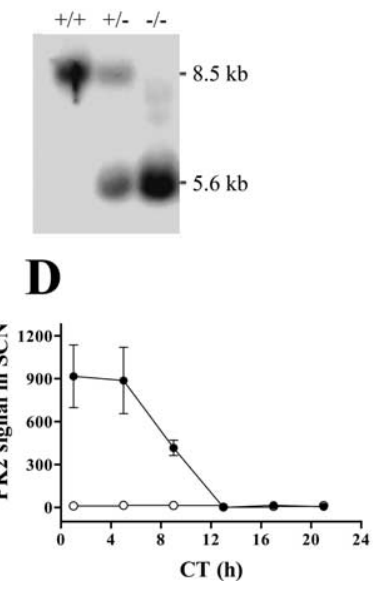

Figure 1. Targeted disruption of the PK2 gene in mice. A, Physical map of the wild-type PK2 locus (top), the targeting vector (middle), and the disrupted PK2 locus (bottom). Exons are indicated by vertical black bars. The $5^{\prime}$ probe used for screening targeted ES clones and mouse genotypes is represented by a gray box. PCR primers used for mouse genotyping are depicted as black arrowheads (P0, P1, and P2, respectively). Luc, Luciferase; Neo, phosphoglycerate kinase promoter-neomycin resistance gene; TK, phosphoglycerate kinase promoter-thymidine kinase gene; B, BamHl; E, EcoRl. B, Identification of targeted mutants by Southern blot hybridization. The $5.6 \mathrm{~kb}$ targeted and the $8.5 \mathrm{~kb}$ wild-type alleles resulting from EcoRl and BamHI digestion are indicated. $C$, Identification of targeted allele by PCR using a three-primer strategy. The 300 bp wild-type allele and $390 \mathrm{bp}$ targeted allele are marked. $\boldsymbol{D}$, In situ hybridization signals of $P K 2$ mRNA in the SCN of wild-type and $P K 2^{-1-}$ mice sampled every $4 \mathrm{~h}$ beginning at $49 \mathrm{~h}$ under constant darkness ( $n=3$ mice; mean \pm SEM). $\boldsymbol{E}$, Representative in situ hybridization images of $P e r 2$ and $P K 2$ mRNAs in the SCN of wild-type and $P K 2^{-1-}$ mice at their respective peak time points.

tary activity) or interruption of infrared beams (e.g., spontaneous activity). We first assessed the circadian rhythm of voluntary locomotor activity with running wheels. $P K 2^{-/-}$mice and their WT littermates were initially entrained to LD for 3 weeks, followed by $4-8$ weeks in DD. In the C57BL/ $6 \times 129 /$ Ola mixed genetic background, all WT and the majority of $P K 2^{-/-}$mice were entrained to the LD and exhibited an identical free-running period under constant darkness (Table 1). However, a small number of $P K 2^{-1-}$ mice ( 4 of 34 ) did not display discernible locomotor rhythm under both LD and DD. The amplitude of locomotor rhythmicity of $P K 2^{-/-}$mice, shown by the relative power of FFT, was significantly reduced under both LD and DD conditions (Table 1). Furthermore, the daily wheel-running counts of $P K 2^{-/-}$mice were significantly lower than that of WT mice under both LD and DD (Table 1). The $P K 2^{-1-}$ mice in the C57BL/6 inbred background consistently showed significantly lower relative FFT power and wheel-running counts than WT mice under both LD and DD (Table 1). One of five $P K 2^{-1-}$ mice in inbred C57BL/6 background was arrhythmic under DD. Furthermore, $P K 2^{-/-}$mice $(\mathrm{C} 57 \mathrm{BL} / 6)$ showed a longer free- running period than WT mice (Table 1 ). Because $P K 2^{-/-}$mice (C57BL/6) displayed very low running counts (supplemental Fig. $\mathrm{S} 1 A$, available at www.jneurosci.org as supplemental material), for comparison of the activity pattern between WT and $P K 2^{-\prime-}$ mice under $\mathrm{LD}$, hourly running counts were normalized to the percentage of $24 \mathrm{~h}$ total activity. As shown in Figure $2 C$, the percentage distribution of wheel-running activity showed significant difference in genotype $\times$ time interaction $\left(F_{(1)}=20.10 ; p<\right.$ $0.0001)$. Especially, the peak activity of $P K 2^{-/-}$mice occurred at the late night compared with early night in the WT mice (Fig. $2 C)$. It should also be noted that $P K 2^{-/-}$mice showed apparently reduced consolidation in the wheel-running activity (Fig. $2 A, B$ ).

We then investigated the spontaneous locomotor activity of $P K 2^{-1-}$ and WT mice using infrared beam recording in their home cages under LD conditions. Consistent with the results from wheel-running behavior, the daily spontaneous activity of $P K 2^{-/-}$mice was significantly lower than WT controls (Table 1). Moreover, the decreased spontaneous activity of $P K 2^{-/-}$mice occurred during the dark period, whereas both genotypes showed similar activity during the light period (supplemental Fig. S1 $B$, available at www.jneurosci.org as supplemental material). Consequently, the ratio of spontaneous locomotor activity observed in the dark compared with the light period was considerably smaller in $P K 2^{-/-}$mice. In addition, a surge of spontaneous activities in $P \mathrm{~K}^{-1-}$ mice also occurred at the late night (Fig. $2 D$ ).

To rule out the possibility that the reduction of the locomotor activity might be the consequence of motor defects, we performed rotarod, hanging-wire, and open-field tests on the $P K 2^{-/-}$mice and their littermate controls. As shown in supplemental Fig. S2 (available at www.jneurosci.org as supplemental material), all of these parameters were indistinguishable between WT and $P K 2^{-/-}$mice, suggesting that motor function was not impaired in $P \mathrm{~K}^{-{ }^{--}}$mice. Together, these results indicate that $P K 2^{-/-}$mice exhibit reduced rhythmicity in both voluntary and spontaneous locomotor activities.

\section{Attenuated circadian rhythms of body temperature and sleep in $P K 2^{-1-}$ mice}

The body temperature in mammals also exhibits a circadian rhythm, which is at least in part controlled by signals from the SCN (Moore, 1997; Schibler and Sassone-Corsi, 2002; Nagashima et al., 2005). The core body temperature is higher in the active phase than inactive phase even under constant darkness. It has been proposed that behavioral or physiological rhythms such as locomotor activity and/or food intake may account for body temperature rhythms. However, the SCN is also able to modulate thermoregulation that results in body temperature rhythm, because the circadian body temperature rhythm persists in completely bed-rested humans, fasting humans and rats, and hibernating squirrels (Nagashima et al., 2005). We implanted body temperature probes (Mini-Mitter) into the abdominal cavities of $P K 2^{-/-}$and WT mice ( $n=5$ mice per group), and the core body temperature was monitored continuously under LD conditions. As shown in Figure $3 A$, both $P K 2^{-/-}$and WT mice showed circadian rhythms in the body temperature. However, the oscillation amplitudes of $P K 2^{-/-}$mice were significantly smaller than WT mice (supplemental Fig. S3, available at www.jneurosci.org as supplemental material). Specifically, the main difference in body temperature occurred during the dark period, when $P K 2^{-1-}$ mice could not reach high peaks as WT mice (Fig. $3 A$ ). The oscillation amplitude of body temperature in $P K 2^{-/-}$mice was also reduced under the DD condition (supplemental Fig. S3 B, C, available at www.jneurosci.org as supplemental material). 


\begin{tabular}{|c|c|c|c|c|c|c|}
\hline & \multicolumn{3}{|c|}{ C57BL/6 × 129/0la } & \multicolumn{3}{|l|}{ C57BL/6 } \\
\hline & Wild-type & $P K 2^{-1-}$ & $p$ value & Wild-type & $P K 2^{-1-}$ & $p$ value \\
\hline Wheel running & $n=30$ & $n=30$ & & $n=10$ & $n=5$ & \\
\hline FFT circadian amplitude (LD) & $0.21 \pm 0.02$ & $0.13 \pm 0.02$ & 0.0008 & $0.22 \pm 0.01$ & $0.04 \pm 0.01$ & $1.08 \times 10^{-5}$ \\
\hline FFT circadian amplitude (DD) & $0.15 \pm 0.02$ & $0.08 \pm 0.01$ & 0.0003 & $0.16 \pm 0.01$ & $0.026 \pm 0.01$ & $2.14 \times 10^{-5}$ \\
\hline Free-running period (h) & $23.65 \pm 0.06$ & $23.65 \pm 0.06$ & 0.93 & $23.43 \pm 0.07$ & $24.22 \pm 0.07$ & 0.0002 \\
\hline Total activity per $24 \mathrm{~h}$ (LD) & $21,441 \pm 2239$ & $13,197 \pm 1739$ & 0.005 & $23,250 \pm 2105$ & $2359 \pm 307$ & 0.00015 \\
\hline Total activity per $24 \mathrm{~h}$ (DD) & $21,321 \pm 2201$ & $11,404 \pm 1729$ & 0.006 & $18,577 \pm 1379$ & $1181 \pm 191$ & $5.28 \times 10^{-6}$ \\
\hline Total spontaneous activity per $24 \mathrm{~h}$ (LD) & $\begin{array}{l}5561 \pm 800 \\
(n=12)\end{array}$ & $\begin{array}{l}2794 \pm 388 \\
(n=12)\end{array}$ & 0.007 & $\begin{array}{l}4417 \pm 662 \\
(n=10)\end{array}$ & $\begin{array}{l}1397 \pm 286 \\
(n=5)\end{array}$ & 0.02 \\
\hline
\end{tabular}

Data are presented as mean \pm SEM. $p$ values represent the results analyzed by Student's $t$ test.

It is well known that SCN plays an important role in circadian regulation of sleep-wakefulness. In rats and mice, SCN lesions affect sleep onset and timing but have no significant effect on either the total amount of sleep or the amount of recovery sleep after sleep deprivation (Mistlberger et al., 1983). In contrast, lesions in the SCN of squirrel monkeys result not only in the loss of sleep circadian rhythm but there is also a $4 \mathrm{~h}$ increase in the amount of sleep, leading to the hypothesis that the output from the SCN enhances wakefulness in diurnal animals (Edgar et al., 1993). The observed attenuation in the rhythmicity of locomotor and the body temperature in $P K 2^{-1-}$ mice may reflect fundamental alterations in the rhythm of sleep-wake cycle (Dijk and Czeisler, 1995; Naylor et al., 2000; Dudley et al., 2003; Shiromani et al., 2004). We directly analyzed the sleep-wake rhythms of $P K 2^{-/-}$and WT mice by implanting EEG and EMG electrodes to monitor the sleep and wake patterns during $2 \mathrm{~d}$ of $\mathrm{LD}$, followed by $2 \mathrm{~d}$ of DD. As shown in Figure $3 B$, the sleep patterns of $P K 2^{-/-}$ and WT mice were significantly different under both LD and DD. Compared with WT mice, $P K 2^{-/-}$mice slept $\sim 85$ min less during each LD cycle (total sleep time: WT, 706.0 $\pm 14.3 \mathrm{~min}$ vs $P K 2^{-/-}, 621.3 \pm 28.2 \mathrm{~min} ; n=11$ mice per group; $p<0.01$ by unpaired $t$ test). Most of the sleep reduction occurred during the light period (total sleep time at light period: WT, $488.0 \pm 8.0 \mathrm{~min}$ vs $P K 2^{-/-}, 423.2 \pm 16.0 \mathrm{~min} ; n=11$ mice per group; $p<0.01$ by unpaired $t$ test) (Fig. $3 C$ ). There was no significant difference in total sleep time between $P K 2^{-/-}$and WT mice during the dark period (WT, $218.0 \pm 12.4 \mathrm{~min}$ vs $P K 2^{-/-}, 198.1 \pm 16.1 \mathrm{~min} ; n=$ 11 mice per group). Because of the reduction of sleep during the light period, the amplitudes of sleep rhythm of $P \mathrm{~K}^{-1-}$ mice were significantly smaller than WT controls under LD, as analyzed by cosine simulation (supplemental Fig. S4A, C, available at www. jneurosci.org as supplemental material). Under DD, the amplitudes of sleep oscillation were also significantly reduced in $P K 2^{-1-}$ mice (supplemental Fig. S4B,C, available at www. jneurosci.org as supplemental material). Thus, PK2 contributes significantly to the magnitude of circadian sleep-wake rhythmicity.

\section{Altered circadian rhythms of circulating corticosterone and glucose levels in $P K 2^{-/-}$mice}

The circadian clock plays an essential role in controlling the daily rhythm of circulating hormones (Moore and Eichler, 1972; Buijs et al., 1993; Buijs and Kalsbeek, 2001; Dubocovich and Markowska, 2005) and energy metabolites (La Fleur et al., 1999; Rudic et al., 2004). In rodents, the plasma corticosterone and glucose levels peak around the onset of the active period (Moore and Eichler, 1972; Buijs et al., 1993; La Fleur et al., 1999). SCN lesion results in the disruption of the glucocorticoid rhythms in rats, specifically the morning glucocorticoid levels are elevated, leading to the hypothesis that SCN output signals function as suppressors for the glucocorticoid level (Moore and Eichler, 1972; Buijs et al., 1993). We investigated the role of PK2 in the circadian regulation of glucocorticoid by measuring the levels of plasma corticosterone in $P K 2^{-/-}$and WT mice at ZT 1 and ZT 11 , time points that approximately correspond to the reported peak and trough times of circulating corticosterone. As shown in Figure $4 A$, WT mice showed a significantly higher corticosterone level at ZT 11 than at ZT 1, whereas there was no significant oscillation of corticosterone level in $P K 2^{-/-}$mice $(p=0.11 ; n=$ $8-10$ mice per time point). Particularly, the $P K 2^{-1-}$ mice had significantly higher corticosterone levels than WT controls at ZT 1 , indicating a possible negative regulation of corticosterone level by PK2 signaling.

Maintaining glucose levels within a narrow range is essential for daily function. Controlled by the SCN, a clear circadian rhythm in blood glucose levels is present (La Fleur et al., 1999; Rudic et al., 2004). Recently, two major clock genes, Clock and Bmall, were shown to be critical for the glucose homeostasis (Rudic et al., 2004). As a target gene of these two transcription factors in the SCN, it is likely that a deficiency in $P K 2$ will affect glucose rhythms. We measured the blood glucose concentrations of $P K 2^{-/-}$and WT mice at approximately peak and trough times, ZT 1 and ZT 11. As shown in Figure $4 B$, glucose level displayed an obvious circadian oscillation in WT mice, with a higher level at ZT 11 than at ZT 1 . However, the oscillation rhythm of blood glucose was abolished in $P K 2^{-/-}$mice.

\section{Normal rhythm of clock genes expression in the SCN of $P K 2^{-1-}$ mice}

The $P K 2^{-1-}$ mice showed identical free-run period as WT mice suggests that PK2 may not be involved in the central clockwork regulation. To further analyze the effect of $P K 2$ deficiency on the clockwork function, we examined the expression of core clockwork genes in the SCN of $P K 2^{-/-}$and WT mice by using in situ hybridization. As shown in Figure 5, the oscillation of all clock genes examined were essentially normal in the SCN of $P K 2^{-/-}$ mice, indicating that loss of the $P K 2$ gene had minimal influence on the function of core clockwork in the SCN. It should also be noted that temporal profiles of vasopressin in the SCN of WT and $P K 2^{-/-}$mice were indistinguishable (Fig. 5). These molecular studies confirm that PK2 acts as an output factor downstream of the core oscillator to regulate circadian rhythms.

\section{Attenuated circadian oscillation in the liver of $P K 2^{-/-}$mice}

Circadian oscillations of clockwork and clock-controlled genes also occur in a variety of peripheral organs. It has been established that the SCN clock is critical in synchronizing these peripheral clocks (Buijs and Kalsbeek, 2001; Reppert and Weaver, 2002; Yoo et al., 2004; Guo et al., 2005). To investigate whether a deficiency in PK2 affects the synchronization of peripheral clocks, we exam- 
A

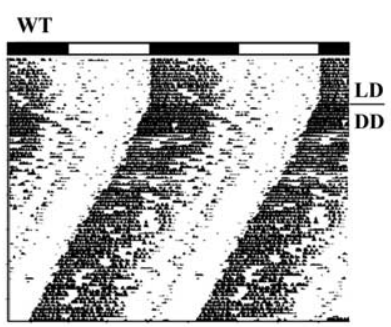

B
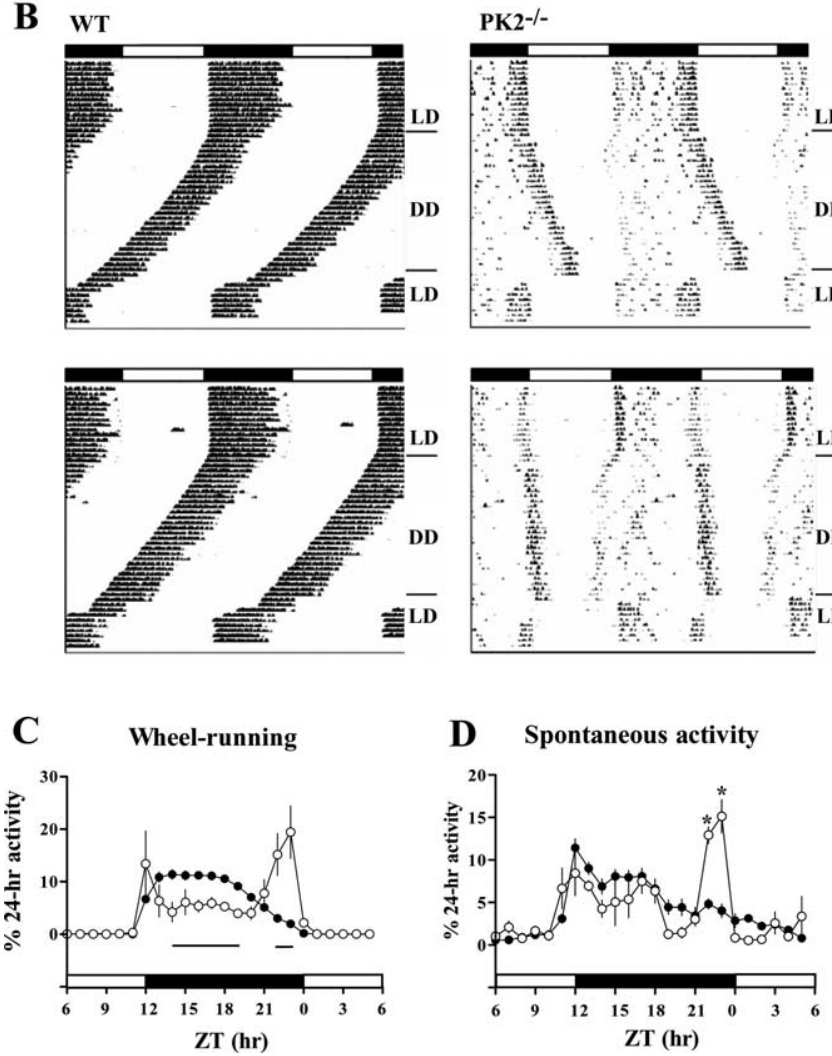

Figure 2. Locomotor activity in WT and $P K 2^{-/-}$mice. $A$, Representative double-plotted wheel-running actograms of a WT (left) and a $P K 2^{-/-}$(right) mouse in the C57BL/6 $\times 129 / 0$ la background. $\boldsymbol{B}$, Representative double-plotted wheel-running actograms of two WT (left) and two $\mathrm{PK2}^{-1-}$ (right) mice in the C57BL/6 background. The bar over the actograms indicates the light/dark cycle. Bin size is $5 \mathrm{~min}$. C, Percentage distribution of wheel-running activity over a $24 \mathrm{~h} \mathrm{LD}$ cycle in WT and $P K 2^{-\prime-}$ mice. For each animal, the wheel running from consecutive $5 \mathrm{~d}$ of $L D$ cycle was accumulated to $1 \mathrm{~h}$ bins and averaged to produce one $24 \mathrm{~h}$ profile. The percentage of each $1 \mathrm{~h}$ activity over $24 \mathrm{~h}$ total activity was calculated and plotted with respect to ZT. Each point represents the mean \pm SEM from five $P K 2^{-1-}$ mice or 10 WT mice in the C57BL/6 background. From ZT 14 to ZT 19 and from ZT 22 to ZT 23 (indicated by the lines underneath the curves), there were significant differences between $P K 2^{-\prime}{ }^{-}$mice and WT mice $(p<0.05$, post hoc Bonferroni's t test). D, Percentage distribution of spontaneous activity over a $24 \mathrm{hLD}$ cycle in WT and $P K 2^{-1-}$ mice (in the C57BL/6 background). There were significant differences between WT and $P K 2^{-1-}$ mice at $Z T 22$ and ZT 23 ( $^{*} p<0.05$, post hoc Bonferroni's $t$ test). WT, Filled circles; $P K 2^{-/-}$, open circles.

ined the molecular oscillation of the clockwork and clockcontrolled genes in the liver, a peripheral organ that does not express detectable $P K 2$ or $P K$ receptor 2 (PKR2) mRNA (data not shown). The mRNA levels of Bmall and Dbp in the liver were measured by real-time PCR. Bmall and Dbp were chosen because they peak at different phases and are regulated by different transcription factors, Rora (RAR-related orphan receptor A) and Bmal1/Clock, respectively (Ripperger et al., 2000; Sato et al., 2004). As shown in Figure 6, $A$ and $B, B m a l 1$ and Dbp displayed circadian rhythms in both WT and $P K 2^{-1-}$ mice. However, the
A
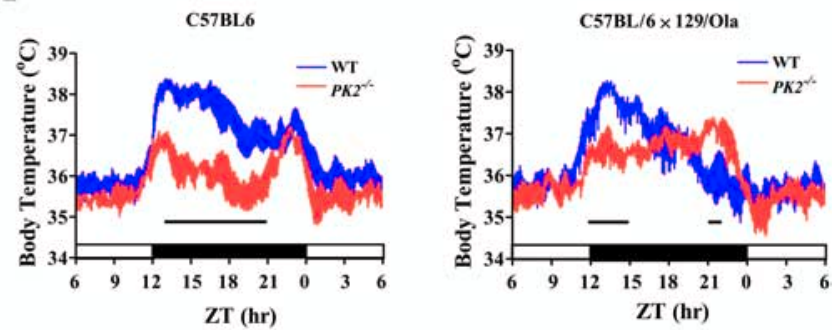

B

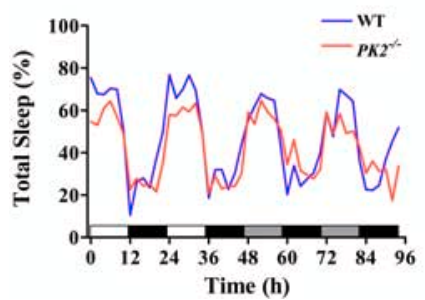

\section{C}

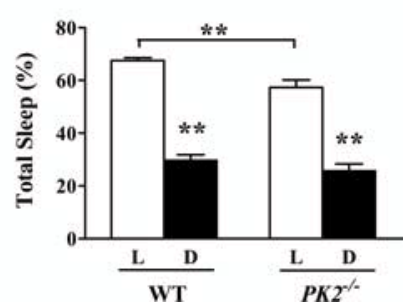

Figure 3. Reduced rhythmicity of core body temperature and sleep-wakefulness in $P K 2^{-1-}$ mice. $A$, The averages of $7 \mathrm{~d}$ recording (5 min bins) of the core body temperature under $\mathrm{LD}$ are plotted. Data are presented as mean \pm SEM from five WT or five $P K 2^{-\prime-}$ mice. In the C57BL/ 6 background (left), there were significant differences between genotypes from ZT 13 to ZT 21 (indicated by the lines underneath the curves; $p<0.05$, post hoc Bonferroni's $t$ test). In the $C 57 \mathrm{BL} / 6 \times 129 / 0$ la background, there were significant difference genotypes from ZT 12 to ZT 15 and from ZT 21 to ZT 22 (indicated by the lines underneath the curves; $p<0.05$, post hoc Bonferroni's $t$ test). $\boldsymbol{B}$, Time course of total sleep for two cycles of $L D$, followed by two cycles of $\mathrm{DD}$. White horizontal bars indicate the light period, black bars indicate the dark periods, and gray bars indicate the subjective light periods under $\mathrm{DD}$. For each animal, total sleep values as percentage of recording time was averaged in $2 \mathrm{~h}$ bins. The data from $11 \mathrm{WT}$ or $11 \mathrm{PK2^{-/- }}$ mice were then averaged and plotted. The total sleep patterns were different between WT and PK2 $2^{-/-}$mice under both $\mathrm{LD}\left(F_{(1)}=25.74 ; p<0.0001\right)$ and $\mathrm{DD}\left(F_{(1)}=11.48 ; p<0.001\right)$. C, Under LD, the total sleep of $P K 2^{-1-}$ mice at light period was significantly reduced compared with WT mice ${ }^{* *} p<0.01$, post hoc Bonferroni's $t$ test). WT, Blue lines; $P K 2^{-1-}$, red lines.
$\mathbf{A}$

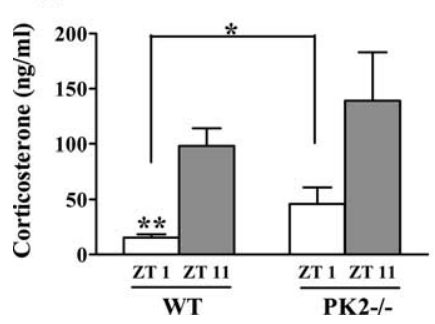

B

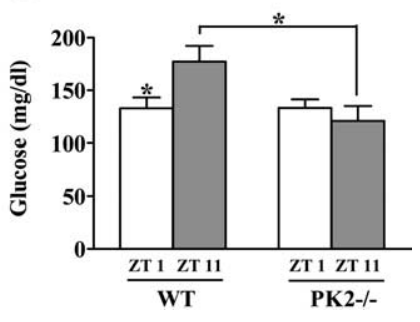

Figure 4. Altered rhythms of circulating corticosterone and glucose levels in $P K 2^{-1-}$ mice. $A$, The circulating corticosterone level at ZT 1 (white columns) of $P K 2^{-1-}$ mice was significantly higher than that of wild-type mice $\left({ }^{*} p<0.05,{ }^{* *} p<0.01\right.$, unpaired $t$ test; $n=8-10$ mice). $B$, Glucose rhythms were significantly different between wild-type and $P K 2^{-1-}$ mice $\left(F_{(1)}=\right.$ $4.41 ; p<0.05 ; n=8-10$ mice). Glucose level at ZT 11 (gray columns) of $P K 2^{-1-}$ mice was significantly lower than that of wild-type mice $\left({ }^{*} p<0.05\right.$, post hoc Bonferroni's $t$ test).

amplitudes of oscillation for Bmal1 and Dbp mRNAs were significantly smaller in $P K 2^{-1-}$ mice, reflected by a $\sim 40 \%$ reduction in the peak levels.

\section{Accelerated adaptation of $P K 2^{-/-}$mice to daytime} restricted feeding

Our observations indicate that the control of SCN over diverse circadian processes is weakened in $P K 2^{-1-}$ mice. To further investigate the deficit in the SCN control of circadian rhythms, we challenged $P K 2^{-/-}$mice with a scheduled daytime RF, which can entrain the circadian rhythms independent of the SCN (Damiola 

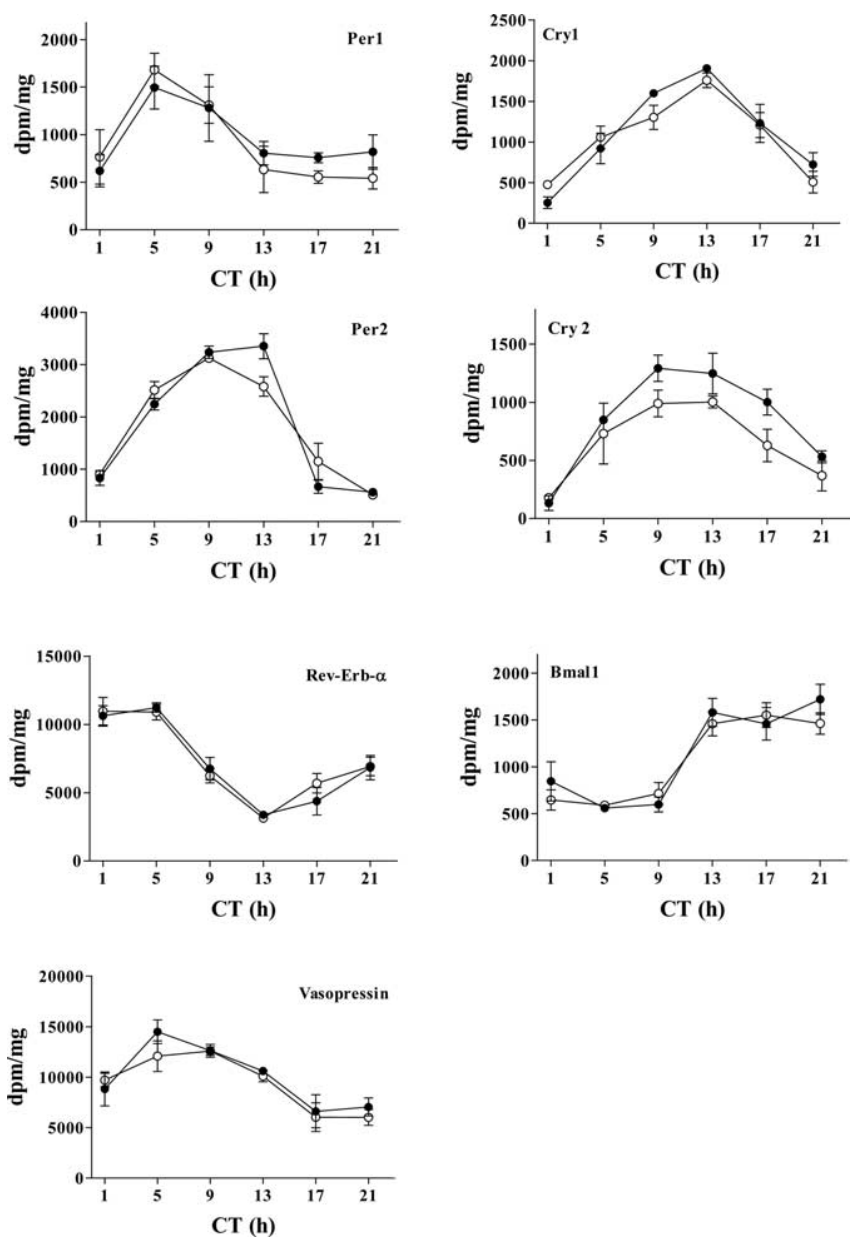

Figure 5. Normal oscillation of clock genes in the $\mathrm{SCN}$ of $P K 2^{-/-}$mice. The $P K 2^{-/-}$mice (open circles) and wild-type controls (filled circles) were killed every $4 \mathrm{~h}$ beginning at $49 \mathrm{~h}$ under constant darkness. In situ hybridizations on coronal brain sections were performed with corresponding ${ }^{35}$ S-labeled probes. No significant difference was detected except Cry2 (crytochrome 2) $\left(F_{(1)}=7.243 ; p=0.013\right)$. Each data represents mean \pm SEM of three mice.
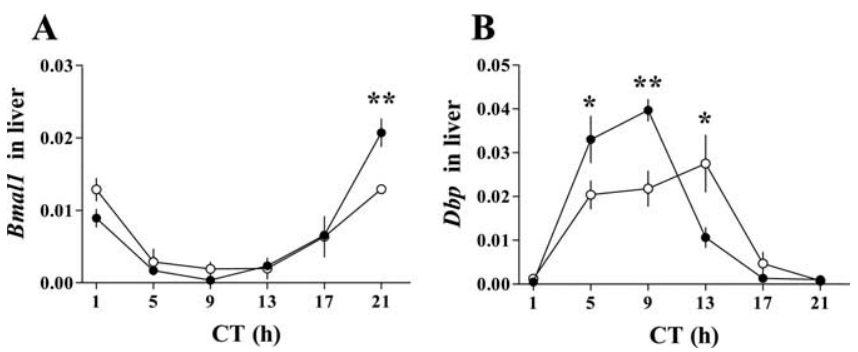

Figure 6. Analysis of clock genes oscillation in the liver of $P K_{2}^{-1-}$ mice. $A$, The circadian rhythm of Bmal1 gene expression in the liver was reduced in $P K 2^{-1-}$ mice $\left(F_{(5)}=4.2 ; p<\right.$ 0.01 , factor genotype $\times$ time). $\boldsymbol{B}$, The circadian rhythm of $D b p$ gene expression in the liver was reduced in $P K 2^{-1-}$ mice $\left(F_{(5)}=8.03 ; p<0.0001\right.$, factor genotype $\times$ time). ${ }^{*} p<0.05$, ${ }^{* *} p<0.01$, genotype difference at time shown by post hoc Bonferroni's $t$ test. WT, Filled circles; PK2 ${ }^{-1-}$, open circles. Each data represents mean \pm SEM of three mice.

et al., 2000; Stokkan et al., 2001). In response to a daytime RF, rodents will feed at unusual period for the sake of survival and gradually become active before the food is made available, a phenomenon called FAA. FAA is an rhythmic event associated with the food entrained oscillators (FEO). During RF, the FEO competes with the light entrained oscillator (LEO) (i.e., SCN) for the control of activity and physiological events. Thus, the higher FAA has been interpreted as a relatively stronger FEO control (Dudley et al., 2003; Pitts et al., 2003). The FAAs of $P K 2^{-/-}$and WT mice were monitored when the food was available between $\mathrm{ZT} 3$ to ZT 7 of LD cycles. As shown in Figure $7, A$ and $B, P K 2^{-1-}$ mice displayed significantly higher FAA during days 3-6 of RF, confirming a weaker SCN control in $P K 2^{-1-}$ mice for the locomotor activity.

\section{Discussion}

During the past decade, studies with mutant animals deficient in clockwork or clock-controlled genes have dramatically advanced our understanding of the circadian clocks. Particularly, these genetic studies have established a molecular frame of interlocked transcriptional and translational loops as the basic operational unit of the SCN circadian clock (Reppert and Weaver, 2002; Hastings and Herzog, 2004; Lowrey and Takahashi, 2004). Our results demonstrated the important role of PK2 for the maintenance of robust circadian rhythms as a SCN output signal. Whereas the majority of $P K 2^{-/-}$mice were still rhythmic, the oscillation amplitudes in multiple circadian parameters were significantly reduced in the absence of the PK2 gene. Parameters that have been analyzed included locomotor activity, sleep-wake cycle, body temperature, circulating glucocorticoid and glucose levels, as well as the expression of peripheral clock genes. The fact that $P K 2^{-/-}$mice had essentially normal clockwork genes oscillation in the SCN suggests that PK2, as an output molecule, has no apparent effect on the core clockwork in the SCN.

Our studies established that one SCN output signal can regulate diverse circadian rhythms, although the abnormal olfactory bulb in $P K 2^{-1-}$ mice may somehow contribute to some of the observed circadian phenotypes (Burge and Edwards, 1976; Possidente et al., 1996; Perret et al., 2003). PK2, as an SCN output signal, may affect various circadian rhythms such as locomotor, sleep-wake, and body temperature via direct or indirect mechanisms. The paraventricular nucleus of the hypothalamus (PVN) is a primary SCN efferent nuclei involved in the direct regulation of endocrine rhythms. Because PKR2 is expressed in the PVN, PK2 may regulate corticosterone level through this pathway. The neurophysiological effect of SCN-derived PK2 on the PVN, one critical SCN target, has been investigated. It has been demonstrated that PK2 was able to excite parvocellular and magnocellular neurons of the PVN (E. A. Yuill, C. C. Ferri, Q. Y. Zhou, and A. V. Ferguson, unpublished data). Importantly, a peptide-based PK2 receptor antagonist was able to decrease the basal activity of parvocellular neurons in the hypothalamus slice (including SCN and PVN) only during light phase, when PK2 is highly expressed in the SCN (Yuill, Ferri, Zhou, and Ferguson, unpublished data). These observations suggest that SCN-derived PK2 may physiologically act as an excitatory signal that increases the firing rate of target neurons.

Although the role of PK2 in synchronizing peripheral oscillators has not been elucidated, the regulatory effect of PK2 is likely to be relayed through secondary or tertiary output signals. Body temperature or circulating factors, such as humoral signals (e.g., glucocorticoid or corticosterone in mice) and metabolites (e.g., glucose), are likely to be synchronizing signals for the oscillators in the peripheral tissues (Buijs and Kalsbeek, 2001; Reppert and Weaver, 2002; Schibler and Sassone-Corsi, 2002). Indeed, $P K 2^{-/-}$mice displayed significantly attenuated oscillation in the core body temperature as well as the levels of circulating corticosterone and glucose, which may account for the reduced oscillation of Bmall and Dbp mRNAs in the liver. The receptor for PK2 is not detectable in the liver, further suggesting that the reduced 
A

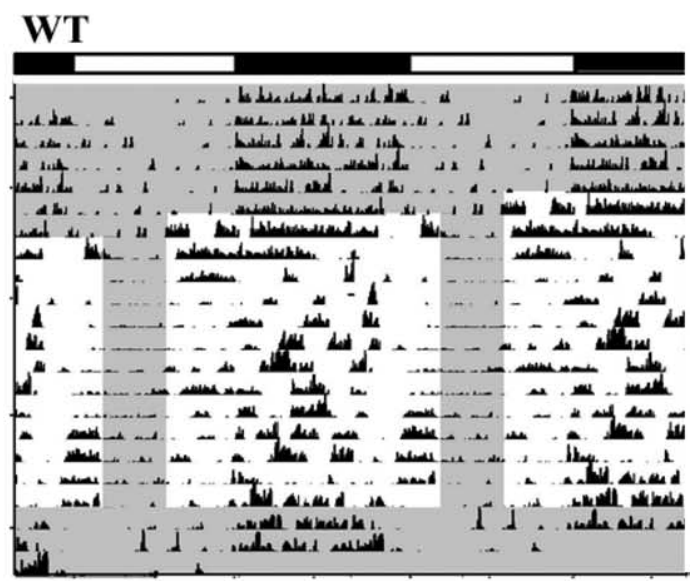

PK2-/-

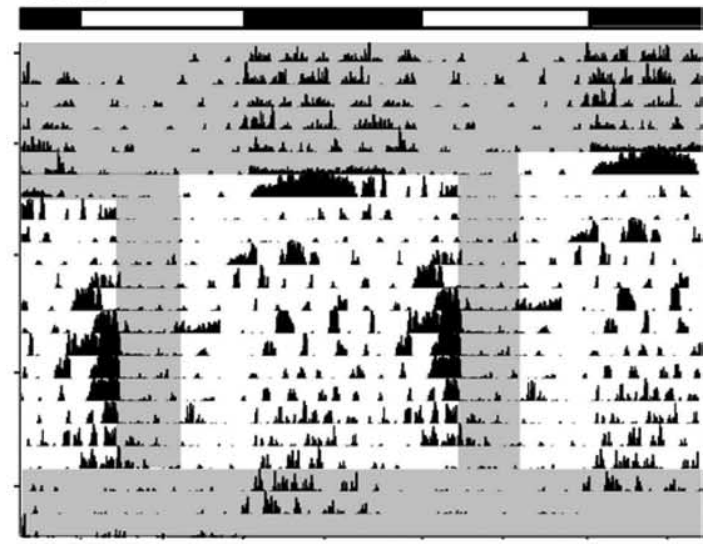

B

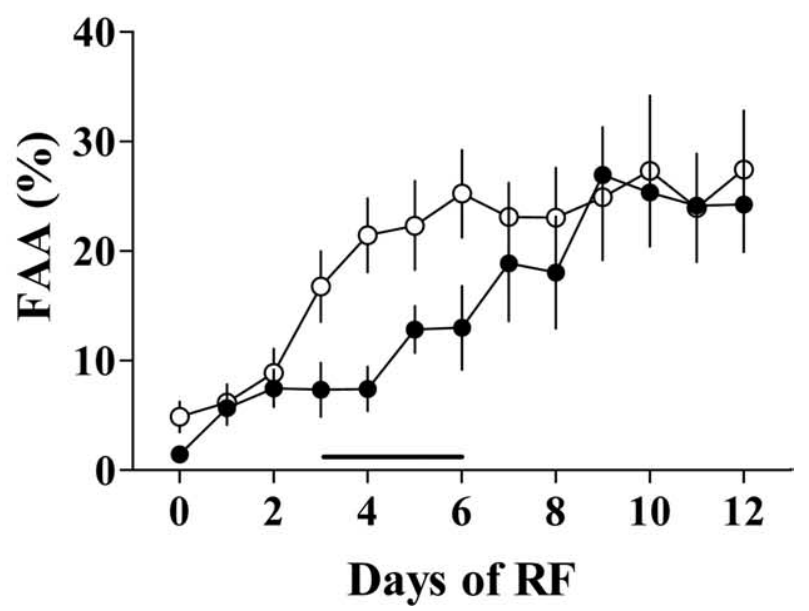

Figure 7. Accelerated adaptation to daytime restricted feeding in $P K 2^{-/-}$mice. $\boldsymbol{A}$, Representative double-plotted actograms of spontaneous locomotor activity in a wild-type mouse (top) and a PK2 $2^{-/-}$mouse (bottom) over $6 \mathrm{~d}$ (days $1-6$ ) of baseline (fed ad libitum) followed by $12 \mathrm{~d}$ (days 7-18) of RF (food only available during ZT 3-7 every day) under LD. The bar over the actograms indicates the LD cycle. Gray shading indicates time during which food was available. $\boldsymbol{B}$, Mean \pm SEM daily amount of FAA during $12 \mathrm{~d}$ of RF. Acquisition of FAA was faster in $P K 2^{-/-}$than wild-type mice $\left(F_{(1)}=9.7 ; p<0.002\right)$. During days $3-6$ of $\mathrm{RF}$ (indicated by the line underneath the curves), the FAA in $P K 2^{-/-}$mice was significantly higher than in WT mice ( $p<0.05$, post hoc Bonferroni's $t$ test). WT, Filled circles; $P K 2^{-1-}$, open circles.

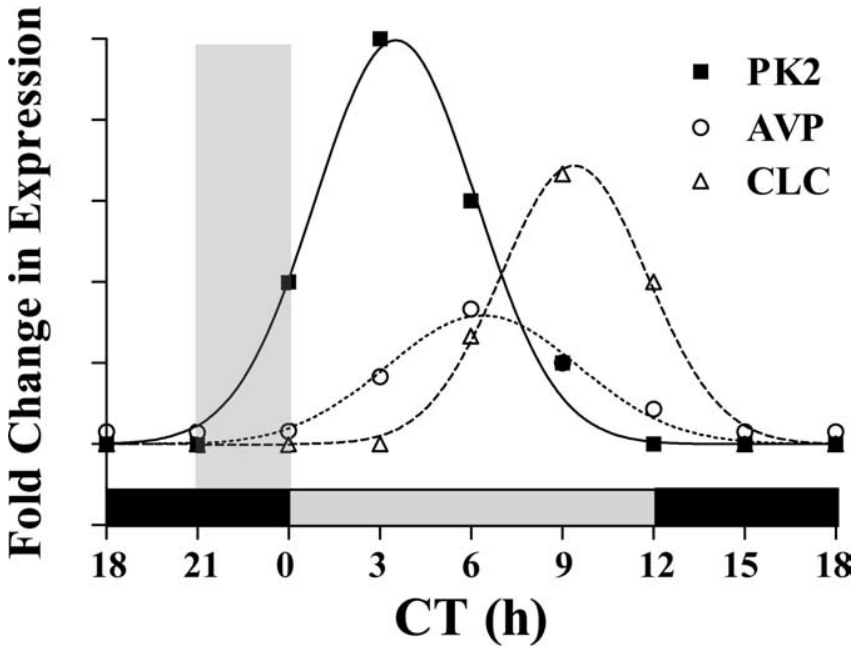

Figure 8. Diagram depicting temporal profiles of $P K 2, A V P$, and $C L C m R N A$ s in the SCN. PK2, $A V P$, and $C L C$ are clock-controlled genes that encode putative secreted SCN output signals. The temporal profiles, including the approximate fold of oscillation, are adopted from Jin et al. (1999), Cheng et al. (2002), and Kraves and Weitz (2006), as well as Figure 5. The temporal oscillation of $P K 2$, AVP, and CLC mRNAs in the SCN shows partial overlapping pattern, with their mRNA levels peaking at different CT of day period: PK2 at approximately CT 3, AVP at approximately CT 6, and CLC at approximately CT 9. Thus, the dawn time (shaded area) represents a specific time zone for the PK2 signal. The gray and black bars indicate the subjective day and night periods, respectively.

liver gene oscillation in $\mathrm{PK}^{-/-}$mice is indirectly regulated through other synchronizing signals. Nevertheless, we cannot totally exclude the possibility that PK2 in peripheral organs, such as gastrointestinal tract, affects the temporal gene expression of the liver.

With regards the sleep-wakefulness (rest-activity) cycle, the regulatory effect of PK2 signal is likely to be indirect; the neuronal activity of the ventrolateral preoptic nucleus and locus ceruleus are regulated by primary SCN efferent nuclei such as dorsomedial hypothalamic nucleus (Aston-Jones et al., 2001; Lu et al., 2002; Chou et al., 2003; Saper et al., 2005), in which PKR2 is highly expressed (Cheng et al., 2002). The timing, duration, and quality of both sleep and wakefulness are regulated by the interaction of two processes, a homeostatic and a circadian timing process (Borbely, 1982; Dijk and Czeisler, 1995). The reduction of sleep time in $P K 2^{-/-}$mice and Clock mutant mice appears to support the concept of a direct molecular link between the homeostatic and circadian regulation of sleep (Naylor et al., 2000; Mignot et al., 2002), as deduced from the sleep studies on the SCN-lesioned squirrel monkeys (Edgar et al., 1993).

Intracerebroventricular infusion of PK2 suppresses the locomotor activity of rats at subjective night (Cheng et al., 2002). However, the general locomotor activity of $P \mathrm{~K}^{-/-}$mice was reduced. Although the definite underlying mechanism for this apparent discrepancy is unclear, it should be noted that the locomotor activity in $P \mathrm{~K}^{-1-}$ mice was relatively elevated during late night, a specific temporal window for PK2 (Figs. $2 B-D, 8$ ). Conversely, it is possible that infusion of PK2 may have limited dispersion such that only nuclei in proximity to the ventricle may have access to PK2, whereas the gene disruption is global. However, we cannot rule out the possible compensatory mechanisms in the global absence of $P K 2$ from a development standpoint. Given the fact that reduced locomotor activity is commonly observed in animals lacking other clock or clock-controlled genes, such as $D B P^{-/-}, V P A C 2^{-/-}$, and $\mathrm{Bmal1}^{-/-}$mutant mice and 
vasopressin-deficient rats (Murphy et al., 1996; Lopez-Molina et al., 1997; Bunger et al., 2000; Harmar et al., 2002), reduced activity might be an exhibition of disturbed circadian rhythms.

The residual circadian rhythms in $P K 2^{-/-}$mice suggest that vasopressin (Jin et al., 1999), CLC (Kraves and Weitz, 2006), and/or other unidentified secreted factors serve as additional SCN output signals. Nevertheless, our results showed that these signals are not sufficient to fully compensate for the loss of PK2 signal. Interestingly, the temporal mRNA profiles of $P K 2$, vasopressin, and CLC in the SCN only partially overlap (Fig. 8). PK2 mRNA peaks at approximately circadian time 3 (CT 3) (Cheng et al., 2002), vasopressin mRNA peaks at approximately CT 6 (Jin et al., 1999), and CLC mRNA peaks at approximately CT 9 (Kraves and Weitz, 2006). Thus, the dawn time represents a unique zone in which the expression of $P K 2$ mRNA does not overlap with vasopressin and CLC. This may explain the increased locomotor activity, wakefulness, body temperature, as well as corticosterone levels in $P K 2^{-/-}$mice during this early morning period (Fig. $2 B, C$, $3 A, 4 A$ ) (supplemental Fig. $S 4 A$, available at www.jneurosci.org as supplemental material) (our unpublished observation). Therefore, SCN-derived PK2 may serve as a suppressive factor for these behavioral and physiological parameters in rodents. Consistent with the expression profiles presented in Figure 8, Kraves and Weitz have shown that blocking CLC signaling by infusion of anti-gp130 caused an increase in locomotor activity specifically during CT 9-CT 12, the peak hours of CLC expression (Kraves and Weitz, 2006).

Among these three putative clock-controlled output molecules, only PK2 and CLC have been shown to modulate the locomotor activity in rodents (Cheng et al., 2002; Kraves and Weitz, 2006). In addition to the $\sim 6 \mathrm{~h}$ difference in the expression peaks, the functional pathways of PK2 and CLC may also be distinct, because their receptors are expressed in different SCN targets. PK2 receptor is expressed in most of the SCN targets but subparaventricular zone, in which receptor for CLC is predominately expressed (Cheng et al., 2002; Kraves and Weitz, 2006). Thus, we propose that, in mammals, PK2 and CLC may gate the locomotor activity in the morning and evening, respectively. This phenomenon coincides evolutionarily with that the morning and evening surges of locomotor activity in the Drosophila melanogaster are driven by distinguished circadian neurons (Stoleru et al., 2004).

Vasopressin has been shown previously as a SCN output signal that suppresses the corticosterone level in rodents (Kalsbeek et al., 1992). Our data indicated that PK2 was also an inhibitory signal for the corticosterone level (Fig. 4A). Recently, PK2 and vasopressin are reported to colocalize partially $(\sim 50 \%)$ in the dorsomedial SCN (Masumoto et al., 2006). Thus, it is likely that PK2 and vasopressin are released to the same SCN targets such as PVN in controlling the corticosterone oscillation, although during different temporal zones. In addition, the signaling pathways of PK2 and vasopressin after binding to their respective receptors (PKR2 and V1aR) also share similarities, including $\mathrm{G}_{\mathrm{q}}$ coupling and the activation of extracellular signal-regulated kinase $1 / 2$ pathway (Birnbaumer, 2000; Lin et al., 2002). CLC is also colocalized with vasopressin in the SCN (Kraves and Weitz, 2006); however, the SCN-derived CLC is unlikely involved in the suppression of corticosterone level, because CLC peaks when corticosterone level is high (Kraves and Weitz, 2006).

The fact that the animals deficient in a given output molecule (vasopressin-deficient rats, $D B P^{-/-}$mice, and $P K 2^{-/-}$mice) display some similar phenotypes, such as attenuated amplitudes of circadian oscillation and reduced locomotor activity (Murphy et al., 1996; Lopez-Molina et al., 1997) indicates a functional redun- dancy among these output factors. The phenomenon that the SCN circadian clock uses multiple output signals, in parallel with the multiple input pathways, reflects the complexity and plasticity of the mammalian circadian clock. It will be intriguing to decipher how these output signals interact in transmitting the SCN timing information to generate overt circadian rhythms.

\section{References}

Aston-Jones G, Chen S, Zhu Y, Oshinsky ML (2001) A neural circuit for circadian regulation of arousal. Nat Neurosci 4:732-738.

Bae K, Jin X, Maywood ES, Hastings MH, Reppert SM, Weaver DR (2001) Differential functions of mPer1, mPer2, and mPer3 in the SCN circadian clock. Neuron 30:525-536.

Birnbaumer M (2000) Vasopressin receptors. Trends Endocrinol Metab $11: 406-410$.

Borbely AA (1982) A two process model of sleep regulation. Hum Neurobiol 1:195-204.

Buijs RM, Kalsbeek A (2001) Hypothalamic integration of central and peripheral clocks. Nat Rev Neurosci 2:521-526.

Buijs RM, Kalsbeek A, van der Woude TP, van Heerikhuize JJ, Shinn S (1993) Suprachiasmatic nucleus lesion increases corticosterone secretion. Am J Physiol 264:R1186-R1192.

Bunger MK, Wilsbacher LD, Moran SM, Clendenin C, Radcliffe LA, Hogenesch JB, Simon MC, Takahashi JS, Bradfield CA (2000) Mop3 is an essential component of the master circadian pacemaker in mammals. Cell 103:1009-1017.

Burge KG, Edwards DA (1976) Olfactory bulb removal results in elevated spontaneous locomotor activity in mice. Physiol Behav 16:83-89.

Cheng MY, Bullock CM, Li C, Lee AG, Bermak JC, Belluzzi J, Weaver DR, Leslie FM, Zhou QY (2002) Prokineticin 2 transmits the behavioural circadian rhythm of the suprachiasmatic nucleus. Nature 417:405-410.

Cheng MY, Bittman EL, Hattar S, Zhou QY (2005) Regulation of prokineticin 2 expression by light and the circadian clock. BMC Neurosci 6:17.

Chou TC, Scammell TE, Gooley JJ, Gaus SE, Saper CB, Lu J (2003) Critical role of dorsomedial hypothalamic nucleus in a wide range of behavioral circadian rhythms. J Neurosci 23:10691-10702.

Damiola F, Le Minh N, Preitner N, Kornmann B, Fleury-Olela F, Schibler U (2000) Restricted feeding uncouples circadian oscillators in peripheral tissues from the central pacemaker in the suprachiasmatic nucleus. Genes Dev 14:2950-2961.

Dijk DJ, Czeisler CA (1995) Contribution of the circadian pacemaker and the sleep homeostat to sleep propensity, sleep structure, electroencephalographic slow waves, and sleep spindle activity in humans. J Neurosci 15:3526-3538.

Dubocovich ML, Markowska M (2005) Functional MT1 and MT2 melatonin receptors in mammals. Endocrine 27:101-110.

Dudley CA, Erbel-Sieler C, Estill SJ, Reick M, Franken P, Pitts S, McKnight SL (2003) Altered patterns of sleep and behavioral adaptability in NPAS2deficient mice. Science 301:379-383.

Edgar DM, Dement WC, Fuller CA (1993) Effect of SCN lesions on sleep in squirrel monkeys: evidence for opponent processes in sleep-wake regulation. J Neurosci 13:1065-1079.

Guo H, Brewer JM, Champhekar A, Harris RB, Bittman EL (2005) Differential control of peripheral circadian rhythms by suprachiasmaticdependent neural signals. Proc Natl Acad Sci USA 102:3111-3116.

Harmar AJ, Marston HM, Shen S, Spratt C, West KM, Sheward WJ, Morrison CF, Dorin JR, Piggins HD, Reubi JC, Kelly JS, Maywood ES, Hastings MH (2002) The VPAC(2) receptor is essential for circadian function in the mouse suprachiasmatic nuclei. Cell 109:497-508.

Hastings MH, Herzog ED (2004) Clock genes, oscillators, and cellular networks in the suprachiasmatic nuclei. J Biol Rhythms 19:400-413.

Jin X, Shearman LP, Weaver DR, Zylka MJ, de Vries GJ, Reppert SM (1999) A molecular mechanism regulating rhythmic output from the suprachiasmatic circadian clock. Cell 96:57-68.

Kalsbeek A, Buijs RM, van Heerikhuize JJ, Arts M, van der Woude TP (1992) Vasopressin-containing neurons of the suprachiasmatic nuclei inhibit corticosterone release. Brain Res 580:62-67.

Kalsbeek A, van Heerikhuize JJ, Wortel J, Buijs RM (1996) A diurnal rhythm of stimulatory input to the hypothalamo-pituitary-adrenal system as revealed by timed intrahypothalamic administration of the vasopressin V1 antagonist. J Neurosci 16:5555-5565.

Kraves S, Weitz CJ (2006) A role for cardiotrophin-like cytokine in the cir- 
cadian control of mammalian locomotor activity. Nat Neurosci 9:212-219.

La Fleur SE, Kalsbeek A, Wortel J, Buijs RM (1999) A suprachiasmatic nucleus generated rhythm in basal glucose concentrations. J Neuroendocrinol 11:643-652.

Lambert CM, Machida KK, Smale L, Nunez AA, Weaver DR (2005) Analysis of the prokineticin 2 system in a diurnal rodent, the unstriped Nile grass rat (Arvicanthis niloticus). J Biol Rhythms 20:206-218.

LeCouter J, Kowalski J, Foster J, Hass P, Zhang Z, Dillard-Telm L, Frantz G, Rangell L, DeGuzman L, Keller GA, Peale F, Gurney A, Hillan KJ, Ferrara $\mathrm{N}$ (2001) Identification of an angiogenic mitogen selective for endocrine gland endothelium. Nature 412:877-884.

Li M, Bullock CM, Knauer DJ, Ehlert FJ, Zhou QY (2001) Identification of two prokineticin cDNAs: recombinant proteins potently contract gastrointestinal smooth muscle. Mol Pharmacol 59:692-698.

Lin DC, Bullock CM, Ehlert FJ, Chen JL, Tian H, Zhou QY (2002) Identification and molecular characterization of two closely related $\mathrm{G}$ proteincoupled receptors activated by prokineticins/endocrine gland vascular endothelial growth factor. J Biol Chem 277:19276-19280.

Lopez-Molina L, Conquet F, Dubois-Dauphin M, Schibler U (1997) The DBP gene is expressed according to a circadian rhythm in the suprachiasmatic nucleus and influences circadian behavior. EMBO J 16:6762-6771.

Lowrey PL, Takahashi JS (2004) Mammalian circadian biology: elucidating genome-wide levels of temporal organization. Annu Rev Genomics Hum Genet 5:407-441.

Lu J, Bjorkum AA, Xu M, Gaus SE, Shiromani PJ, Saper CB (2002) Selective activation of the extended ventrolateral preoptic nucleus during rapid eye movement sleep. J Neurosci 22:4568-4576.

Masumoto KH, Nagano M, Takashima N, Hayasaka N, Hiyama H, Matsumoto S, Inouye ST, Shigeyoshi Y (2006) Distinct localization of prokineticin 2 and prokineticin receptor 2 mRNAs in the rat suprachiasmatic nucleus. Eur J Neurosci 23:2959-2970.

Mignot E, Taheri S, Nishino S (2002) Sleeping with the hypothalamus: emerging therapeutic targets for sleep disorders. Nat Neurosci [Suppl] 5:1071-1075.

Mistlberger RE, Bergmann BM, Waldenar W, Rechtschaffen A (1983) Recovery sleep following sleep deprivation in intact and suprachiasmatic nuclei-lesioned rats. Sleep 6:217-233.

Moore RY (1997) Circadian rhythms: basic neurobiology and clinical applications. Annu Rev Med 48:253-266.

Moore RY, Eichler VB (1972) Loss of a circadian adrenal corticosterone rhythm following suprachiasmatic lesions in the rat. Brain Res 42:201-206.

Murphy HM, Wideman CH, Nadzam GR (1996) The interaction of vasopressin and the photic oscillator in circadian rhythms. Peptides 17:467-475.

Nagashima K, Matsue K, Konishi M, Iidaka C, Miyazaki K, Ishida N, Kanosue $\mathrm{K}$ (2005) The involvement of Cryl and Cry2 genes in the regulation of the circadian body temperature rhythm in mice. Am J Physiol Regul Integr Comp Physiol 288:R329-R335.

Naylor E, Bergmann BM, Krauski K, Zee PC, Takahashi JS, Vitaterna MH,
Turek FW (2000) The circadian clock mutation alters sleep homeostasis in the mouse. J Neurosci 20:8138-8143.

Ng KL, Li JD, Cheng MY, Leslie FM, Lee AG, Zhou QY (2005) Dependence of olfactory bulb neurogenesis on prokineticin 2 signaling. Science 308:1923-1927.

Perret M, Aujard F, Seguy M, Schilling A (2003) Olfactory bulbectomy modifies photic entrainment and circadian rhythms of body temperature and locomotor activity in a nocturnal primate. J Biol Rhythms 18:392-401.

Pitts S, Perone E, Silver R (2003) Food-entrained circadian rhythms are sustained in arrhythmic Clk/Clk mutant mice. Am J Physiol Regul Integr Comp Physiol 285:R57-R67.

Possidente B, Lumia AR, McGinnis MY, Rapp M, McEldowney S (1996) Effects of fluoxetine and olfactory bulbectomy on mouse circadian activity rhythms. Brain Res 713:108-113.

Reppert SM, Weaver DR (2002) Coordination of circadian timing in mammals. Nature 418:935-941.

Ripperger JA, Shearman LP, Reppert SM, Schibler U (2000) CLOCK, an essential pacemaker component, controls expression of the circadian transcription factor DBP. Genes Dev 14:679-689.

Rudic RD, McNamara P, Curtis AM, Boston RC, Panda S, Hogenesch JB, Fitzgerald GA (2004) BMAL1 and CLOCK, two essential components of the circadian clock, are involved in glucose homeostasis. PLoS Biol 2:e377.

Saper CB, Scammell TE, Lu J (2005) Hypothalamic regulation of sleep and circadian rhythms. Nature 437:1257-1263.

Sato TK, Panda S, Miraglia LJ, Reyes TM, Rudic RD, McNamara P, Naik KA, FitzGerald GA, Kay SA, Hogenesch JB (2004) A functional genomics strategy reveals Rora as a component of the mammalian circadian clock. Neuron 43:527-537.

Schibler U, Sassone-Corsi P (2002) A web of circadian pacemakers. Cell 111:919-922.

Shiromani PJ, Xu M, Winston EM, Shiromani SN, Gerashchenko D, Weaver DR (2004) Sleep rhythmicity and homeostasis in mice with targeted disruption of mPeriod genes. Am J Physiol Regul Integr Comp Physiol 287:R47-R57.

Stokkan KA, Yamazaki S, Tei H, Sakaki Y, Menaker M (2001) Entrainment of the circadian clock in the liver by feeding. Science 291:490-493.

Stoleru D, Peng Y, Agosto J, Rosbash M (2004) Coupled oscillators control morning and evening locomotor behaviour of Drosophila. Nature 431:862-868.

Watts AG, Swanson LW (1987) Efferent projections of the suprachiasmatic nucleus. II. Studies using retrograde transport of fluorescent dyes and simultaneous peptide immunohistochemistry in the rat. J Comp Neurol 258:230-252.

Watts AG, Swanson LW, Sanchez-Watts G (1987) Efferent projections of the suprachiasmatic nucleus. I. Studies using anterograde transport of Phaseolus vulgaris leucoagglutinin in the rat. J Comp Neurol 258:204-229.

Yoo SH, Yamazaki S, Lowrey PL, Shimomura K, Ko CH, Buhr ED, Siepka SM, Hong HK, Oh WJ, Yoo OJ, Menaker M, Takahashi JS (2004) PERIOD2::LUCIFERASE real-time reporting of circadian dynamics reveals persistent circadian oscillations in mouse peripheral tissues. Proc Natl Acad Sci USA 101:5339-5346. 